\title{
Highly Efficient, ITO-Free Polymer Solar Cells Using Ultrathin Copper Film as Transparent Electrode
}

\author{
Quanbin Liang ${ }^{1}$, Guoping Luo ${ }^{1,2, *}$, Xiaoping Cheng ${ }^{1}$, Hongbin $\mathrm{Wu}^{1, *}$ \\ 1 Institute of Polymer Optoelectronic Materials and Devices, State Key Laboratory of Luminescent Materials \\ and Devices, South China University of Technology, Guangzhou 510640, P. R. China;liangqb@scut.edu.cn \\ (Q.L.); $\underline{52565900 @ q q . c o m}(X . C$. \\ 2 College of Science, Guangdong University of Petrochemical Technology, Maoming 525000, P. R. China; \\ *Correspondence: gpluo@gdupt.edu.cn (G.L.); hbwu@scut.edu.cn (H.W.);Tel.: +86-020-22237016
}

\begin{abstract}
Here we report highly efficient, indium tin oxide (ITO)-free polymer solar cells (PSCs) with an ultrathin copper $(\mathrm{Cu})$ film $(\sim 10 \quad \mathrm{~nm})$ coated with a thin layer of poly[(9,9-bis(3'-(N,N-dimethylamino)propyl)-2,7-fluorene)-alt-2,7-(9,9-dioctylfluorene)] (PFN) as transparent electrode. Despite of its lower far-field transmittance of the electrode, the obtained ITO-free device based on the ultrathin $\mathrm{Cu}$ film can delivery higher absorption efficiency than that of the device based on ITO substrate in the long wavelength region, which can be attributed to the formation of metal resonant microcavity between the opaque back metal mirror $\left(\mathrm{MoO}_{3} / \mathrm{Al}\right.$ electrode) and the transparent $\mathrm{Cu}$ film with high reflectance. As a result, polymer solar cells based on

poly[[2,6'-4,8-di(5-ethylhexylthienyl)benzo[1,2-b;3,3-b]dithiophene][3-fluoro-2[(2-ethylhexyl)carbo nyl]thieno[3,4-b]thiophenediyl]] (PTB7-Th) and [6,6]-phenyl C71-butyric acid methyl ester (PC $71 \mathrm{BM})$ blend show a high power conversion efficiency (PCE) of $8.21 \%$, comparable to that of the control device based on ITO electrode (with a PCE of $9.60 \%$ ). The results demonstrate that thermally evaporated $\mathrm{Cu}$ thin film electrode can be promising candidate to replace ITO for highly efficient PSCs, thus may open up the possibility for massive production of PSCs with low cost.
\end{abstract}

Keywords: ITO-free polymer solar cells; resonant microcavity; ultrathin $\mathrm{Cu}$ film

\section{Introduction}

Polymer solar cells (PSCs) have potential to become the next-generation low-cost renewable energy source, because of their unique advantages such as abundant material resources, solution-based processing technique, and the compatibility with lightweight, flexible substrates and roll-to-roll manufacturing[1]. Over the past two decades, the power conversion efficiencies (PCEs) of state-of-the-art PSCs have been rapidly enhanced owing to the efforts in the design and synthesis of novel materials, especially the emerging non-fullerene acceptor materials[2-4] and optimization in active layer morphology and device structure[5]. To date, the best certified PCEs in the scientific literatures have reached $16 \%$ in single-junction PSCs[6] and even higher values of $17 \%$ in tandem solar cells[7]. Nevertheless, most of the reported efficient PSCs rely on indium tin oxide (ITO) as transparent electrode, mainly due to its high optical transparency ( $>85 \%$ in the visible spectrum), high conductivity $(4000 \mathrm{~S} / \mathrm{cm})$ and its capacity for being finely etched. However, indium is a rare metal on the earth and is very expensive, thus limits their applications for mass production. Therefore, replacing ITO transparent electrodes with cheaper alternatives for PSCs is in urgent need in order to meet the requirement for low-cost organic photovoltaic devices. In recent years, tremendous efforts have been shifted to many emerging transparent electrode materials, including conducting oxides[8], metal nanowires [9,10], metal grids[11]and thin metal films (TMFs) [12,13]. Among a great variety of novel TMFs, Ag is the most widely used metal, with excellent optical and electrical properties. Furthermore, the far-field transparency of Ag films can be greatly improved through anti-reflective coating by using a triple-layer (sandwich architecture with seed layer/metal 
film/interfacial layer) electrode structure [14], where the seed layer can be sulfide [15], metal oxide [16], small-molecule[16] and even polymer[17]. However, in order to obtain a fine Ag thin film, the treatment of substrate and seed layer is complicated. Moreover, the deposition environment and conditions must be rigorously controlled. Therefore, a simplified electrode fabrication process and replacement of silver by a much cheaper metal are highly desired. For example, Li et al. reported the use of printed copper film as the bottom electrode for flexible PSCs [18]. In addition, the use of ultra-thin $\mathrm{Cu}$ and tungsten suboxide (WO3-x) bilayer window electrodes as cathode for organic photovoltaic was also demonstrated by Hutter et al.[19]. More recently we applied a bilayer electrode consisting of a 15-nm $\mathrm{Cu}$ thin layer and poly[(9,9-bis(3'-(N,N-dimethylamino)propyl)-2,7-fluorene)-alt-2,7-(9,9-dioctylfluorene)](PFN)

interlayer for polymer solar cells based on poly[N-9"'-hepta-decanyl-2,7-carbazole-alt-5,5-(4',7'-di-2-thienyl-2', $1^{\prime}, 3^{\prime}$-benzothiadiazole)(PCDTBT ). Nevertheless, the resulted devices only showed a moderate PCE of $3.6 \%$, mainly due to wide bandgap of PCDTBT (with an absorption onset of $\sim 650 \mathrm{~nm}$ ) and the too low far-field optical transmittance of the $15 \mathrm{~nm} \mathrm{Cu}$ thin layer in the long wavelength region. Thus, the use of promising low bandgap donors that with extended the spectral responsitivity to the near infrared (NIR) region should be able to fully exploit the potential of ultrathin metal layer as promising transparent electrode for ITO-free PSCs.

Here we report the fabrication of highly efficient, ITO-free polymer solar cells based on low bandgap electron donors, in which ultrathin copper film $(10 \mathrm{~nm})$ was employed as the transparent electrode, without the need of any seed layers. The metal electrode was spin-coated with a thin layer of PFN as the cathode interfacial layer and the devices were fabricated in an inverted type structure. The bilayer electrode shows high optical transmittance in both visible region and the NIR region, which is crucial to achieve high PCE in PSCs from low bandgap materials. With this ultrathin copper film $(10 \mathrm{~nm})$ as transparent, reflective metal electrode and poly[[2,6'-4,8-di(5-ethylhexylthienyl)benzo[1,2-b;3,3-b]dithiophene][3-fluoro-2[(2-ethylhexyl)carbon yl]thieno[3,4-b]thiophenediyl]](PTB7-Th) and [6,6]-phenyl C71-butyric acid methyl ester (PC71BM) blend as the photoactive layer, a remarkable PCE of $8.21 \%$ was achieved. Moreover, as compared with the device based on ITO substrate, the obtained ITO-free device delivery higher photoresponse in the long wavelength region, which can be attributed to the formation of metal resonant microcavity that can enhance light absorption in the photoactive layer. Because of the ease of fabrication, low-cost and comparable device performance provided by this simple metal thin film based electrode, we anticipated it can be used to fully exploiting the potential of various material system, especially those low bandgap materials, toward a more cost-effective, and ITO-free PSCs or NIR photodetectors.

\section{Experimental Section}

\subsection{Materials and device Fabrication}

Electron donor polymer PTB7-Thwere purchased from 1-material Inc. (Chemin St-Francois, Dorval, Quebec, Canada), while PC ${ }_{71} \mathrm{BM}$ was purchased from Luminescent Technology Inc. The interlayer PFN was synthesized in the lab following literature[20]. Device preparation and characterization were carried out in clean room conditions. The blank glasses (Zhuhai Kaivo Optoelectronic Technology CO., Ltd, Zhuhai, China) and ITO substrates (CSG Holding Co., Ltd., Shenzhen, China) were sequentially cleaned with acetone, detergent, de-ionized water, and isopropyl alcohol. After drying, the glass substrates were transferred into vacuum chamber. $\mathrm{Cu}$ films were deposited by thermal evaporation at a rate of $1 \AA \mathrm{s}^{-1}$ under vacuum pressure $<10^{-4} \mathrm{~Pa}$. After thermal evaporation, a thin layer of PFN $(\sim 10 \mathrm{~nm})$ was spin-coated onto the $\mathrm{Cu}$ films or ITO substrate from methanol solution $(0.5 \mathrm{mg} / \mathrm{ml})$. Subsequently, the photoactive layer solution was deposited on top of the PFN layer via spin coating. The PTB7-Th:PC ${ }_{71} \mathrm{BM}$ blends solution was prepared following literature[21].Finally, molybdenum oxide $(\sim 10 \mathrm{~nm})$ and aluminum $(\sim 100 \mathrm{~nm})$ was thermally evaporated onto the active layer sequentially under vacuum pressure $<10^{-4} \mathrm{~Pa}$. All the 
fabricated solar cells were encapsulated using epoxy inside a glovebox. All of the processes were carried out in a controlled atmosphere of a nitrogen dry-box containing less than 10 ppm oxygen and moisture except for the cleaning of substrate.

\subsection{Characterization and Measurement}

The optical transmittance was measured using a UV-VIS-NIR spectrophotometer (Shimadzu UV-3600). The sheet resistance of $\mathrm{Cu}$ films and ITO substrate were measured in a four-point probe setup. The refractive index $(n)$ and extinction coefficient $(k)$ of active layers were measured by using a VASE ellipsometer from J. A.Woollam Co., Inc. The values of power conversion efficiency were determined from $J-V$ characteristics measured by a Keithley 2400 source-measurement unit under AM 1.5 G spectrum from a solar simulator (SAN-EI, XES-40S1 150W AAA). The active area of device was defined by an electrode mask and determined as $16.0 \mathrm{~mm}^{2}$. Solar simulator illumination intensity was determined by using a mono-crystal silicon reference cell (Hamamatsu S1133, with KG-5 visible color filter), which was calibrated by the National Renewable Energy Laboratory (NREL). External quantum efficiencies and device reflection were measured by an integrated system (QE-R, Enlitech Inc.).

\section{Results and Discussion}

\subsection{Photovoltaic Performance}

Previously we demonstrated that by tuning the thickness of ultrathin copper $(\mathrm{Cu})$ film, optimized overall electrical and optical properties can be obtained when the thickness was fixed at $15 \mathrm{~nm}$ [22]. However, the optical transmittance of the $15 \mathrm{~nm} \mathrm{Cu}$ film is not high enough to ensure maximal absorption in the active layer from low bandgap donor materials, especially for those donors with intense absorption in long wavelength region $(>600 \mathrm{~nm})$. To fully exploiting the potential of the $\mathrm{Cu}$ thin film based electrode for various material systems, a practical approach is using thinner $\mathrm{Cu}$ film (i.e. $<10 \mathrm{~nm}$ ) as electrode, despite ultrathin $\mathrm{Cu}$ film is very sensitive to in-situ oxidation. Nevertheless, with the cover of PFN thin layer atop, the $\mathrm{Cu}(10 \mathrm{~nm}) / \mathrm{PFN}(10 \mathrm{~nm})$ bilayer film show a maximal transmittance of $76 \%$ (at $600 \mathrm{~nm}$ ) (see Figure.1), while a typical ITO (130 $\mathrm{nm}) / \mathrm{PFN}(10 \mathrm{~nm}$ ) electrode for inverted devices shows a maximal transmittance of $87 \%$ (at $489 \mathrm{~nm}$ ). More importantly, the bilayer film has a high average transmittance around $70 \%$ in the long wavelength region between 600 and $800 \mathrm{~nm}$, which is important for efficient PSCs based on narrow bandgap materials. Furthermore, despite the ultrathin $\mathrm{Cu}$ film show a gradual decreases in the transmittance in the long wavelength $(>600 \mathrm{~nm})$, where the near infrared (NIR) reflection begins to dominate the spectral behavior, however, this reduction in transmittance of electrode does not always imply reduced absorption in a solar cell[13]. 


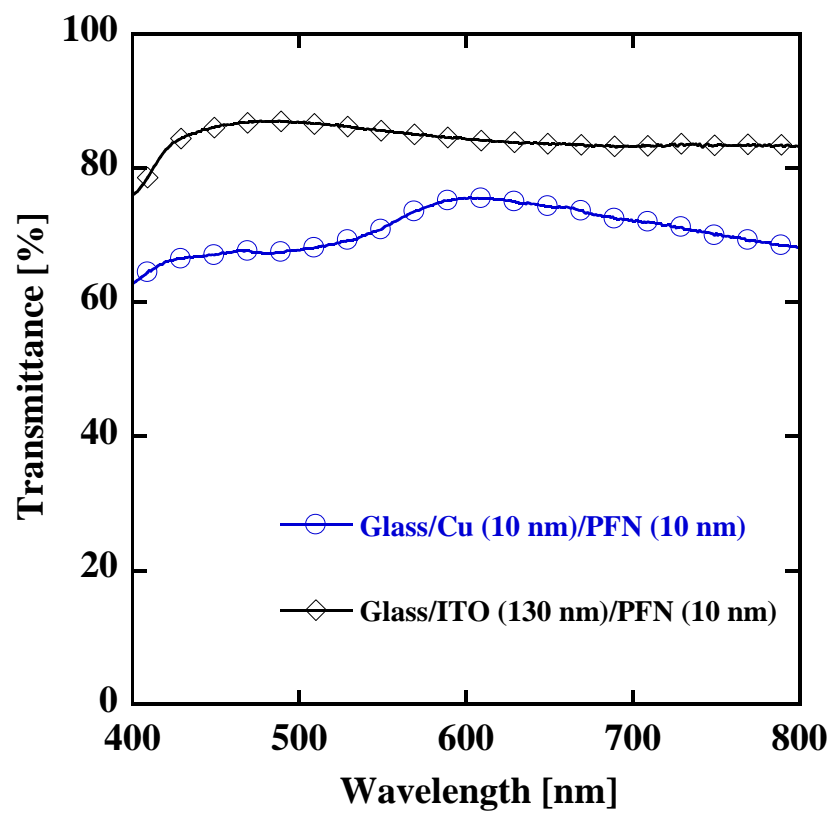

Figure 1.The transmittance spectra of the $\mathrm{Cu}(10 \mathrm{~nm}) / \mathrm{PFN}(10 \mathrm{~nm})$ bilayer electrode. The spectra of an ITO-based reference electrode for inverted PSCs are also included for comparison.

Therefore, combined with appropriate work function $(\sim 4.0 \mathrm{eV})$, the $\mathrm{Cu}(10 \mathrm{~nm}) / \mathrm{PFN}(10 \mathrm{~nm})$ bilayer film can be utilized as electrode candidate for efficient, inverted typed, ITO-free PSCs that have strong photoresponse in $600-800 \mathrm{~nm}$. To verify the validity of the films for efficient PSCs, polymer PTB7-Th with narrow-bandgap $(\sim 1.6 \mathrm{eV})$ was employed as the electron donor materials, which showed a strong absorption band between $600 \mathrm{~nm}$ and $780 \mathrm{~nm}$ [21]. The current density versus voltage $(J-V)$ characteristics of the best PTB7-Th: PC ${ }_{71}$ BMdevices based on the $\mathrm{Cu}(10 \mathrm{~nm}) / \mathrm{PFN}(10$ $\mathrm{nm}$ ) bilayer film as tested under $100 \mathrm{~mW} \mathrm{~cm}^{-2}$ air mass 1.5 global (AM 1.5G) illumination were depicted in Figure.2. And the corresponding parameters of these devices are shown in Table 1. Device performance parameters such as $\mathrm{V}_{\mathrm{oc}}, J_{s c}$, and $\mathrm{FF}$ that deduced from the $J-V$ characteristics are $0.79 \mathrm{~V}, 15.2 \mathrm{~mA} \mathrm{~cm}^{-2}$ and $68.2 \%$, respectively. The overall PCE is found to be $8.21 \%$. A control device based on ITO/PFN electrode shows a PCE of $9.60 \%$ (with a $V_{\text {oc }}$ of $0.79 \mathrm{~V}$, a $J s c$ of $17.5 \mathrm{~mA} \mathrm{~cm}^{-2}$, and a FF of $69.6 \%$, respectively), which is consistent with our previous report[21]. Therefore, the ITO-free devices reported here can maintain an high efficiency of around $85 \%$ as that of the control device, which is much higher than that of our previous results $(\sim 72 \%)$ [22], suggesting that the reduction in the thickness of the $\mathrm{Cu}$ ultrathin film and the use of narrow bandgap semiconducting polymer are the key factors towards these highly efficient ITO-free devices.

Table 1. The photovoltaic parameters of $\mathrm{Cu} / \mathrm{PFN}$ device and ITO device.

\begin{tabular}{ccccc}
\hline Electrode & $\begin{array}{c}\mathbf{J}_{s c} \\
\left(\mathbf{m A} / \mathbf{c m}^{2}\right)\end{array}$ & $\begin{array}{c}\mathbf{V}_{\text {oc }} \\
(\mathbf{V})\end{array}$ & FF & $\begin{array}{c}\text { PCE } \\
\mathbf{( \% )}\end{array}$ \\
\hline Cu/PFN & 15.2 & 0.79 & 0.68 & 8.21 \\
ITO & 17.5 & 0.79 & 0.69 & 9.60 \\
\hline
\end{tabular}




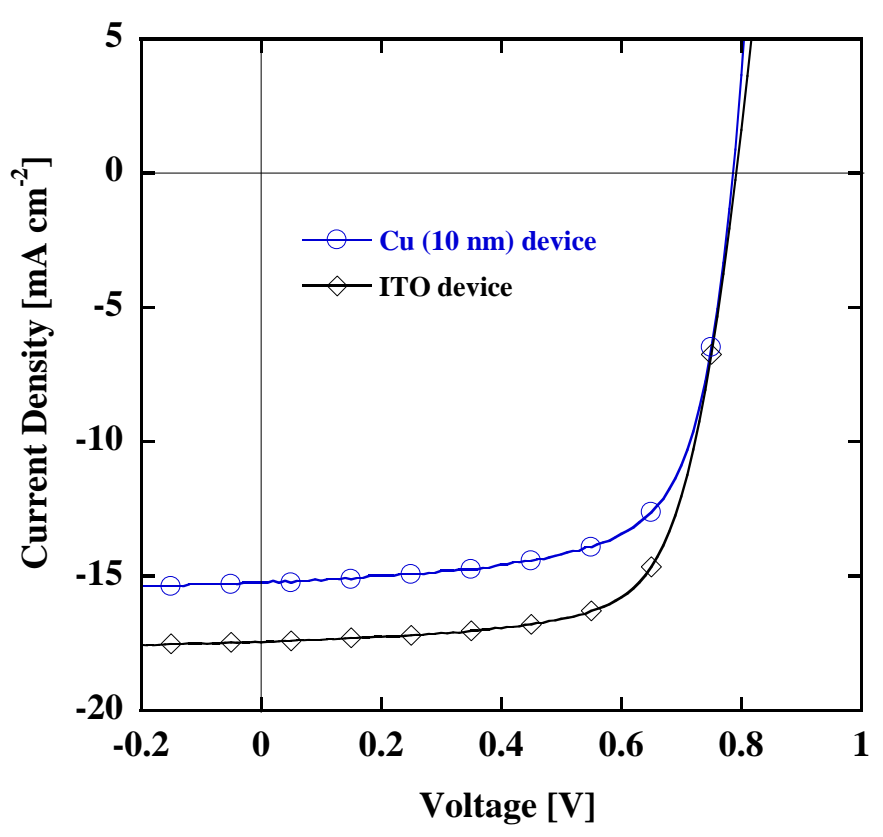

Figure 2.The current density-voltage $(J-V)$ characteristics for the $\mathrm{Cu}$ electrode and ITO electrode devices under AM 1.5 G illumination.

\subsection{Optical resonant microcavity effect}

Efficient photons harvest in the ITO-free devices based on the ultrathin $\mathrm{Cu}$ film can be further evidenced by the optical modeling result, in which one-dimensional transfer matrix formalism was used to calculate the light absorption profile inside the active layer[23]. As shown in Figure.3a, the average absorbed incident photon flux density in $\mathrm{Cu}(10 \mathrm{~nm})$ devices is only $10 \%$ less than that of the ITO devices, obviously higher that the $\mathrm{Cu}(15 \mathrm{~nm}$ ) devices (which is $\sim 20 \%$ less).Furthermore, it can be seen from Figure.3b, the calculated $J s c$ in the $\mathrm{Cu}(10 \mathrm{~nm})$ devices are always higher than that of the $\mathrm{Cu}(15 \mathrm{~nm})$ devices, revealing that reduction in the thickness of the $\mathrm{Cu}$ ultrathin film is indeed one of the key factor toward high efficiency in these devices. Nevertheless, the calculated Jsc values in both $\mathrm{Cu}$ films devices are lower than the ITO devices. On the other hand, at the optimized active layer thickness (which is determined as $\sim 80-100 \mathrm{~nm}$ for this PTB7-Th: $\mathrm{PC}_{71} \mathrm{BM}$ system), the $\mathrm{Cu}(10 \mathrm{~nm})$ devices and the ITO devices shows similar calculated $J s c$ values $\left(17.2 \mathrm{~mA} \mathrm{~cm}-2 \mathrm{vs} .18 .5 \mathrm{~mA} \mathrm{~cm}^{-2}\right)$. It is worthy to mention that the calculated $J s c$ values were obtained on the basis of an ideal situation (with an internal quantum efficiency of $100 \%$ ), thus the calculated Jsc values are found to be slightly higher than measured values. 

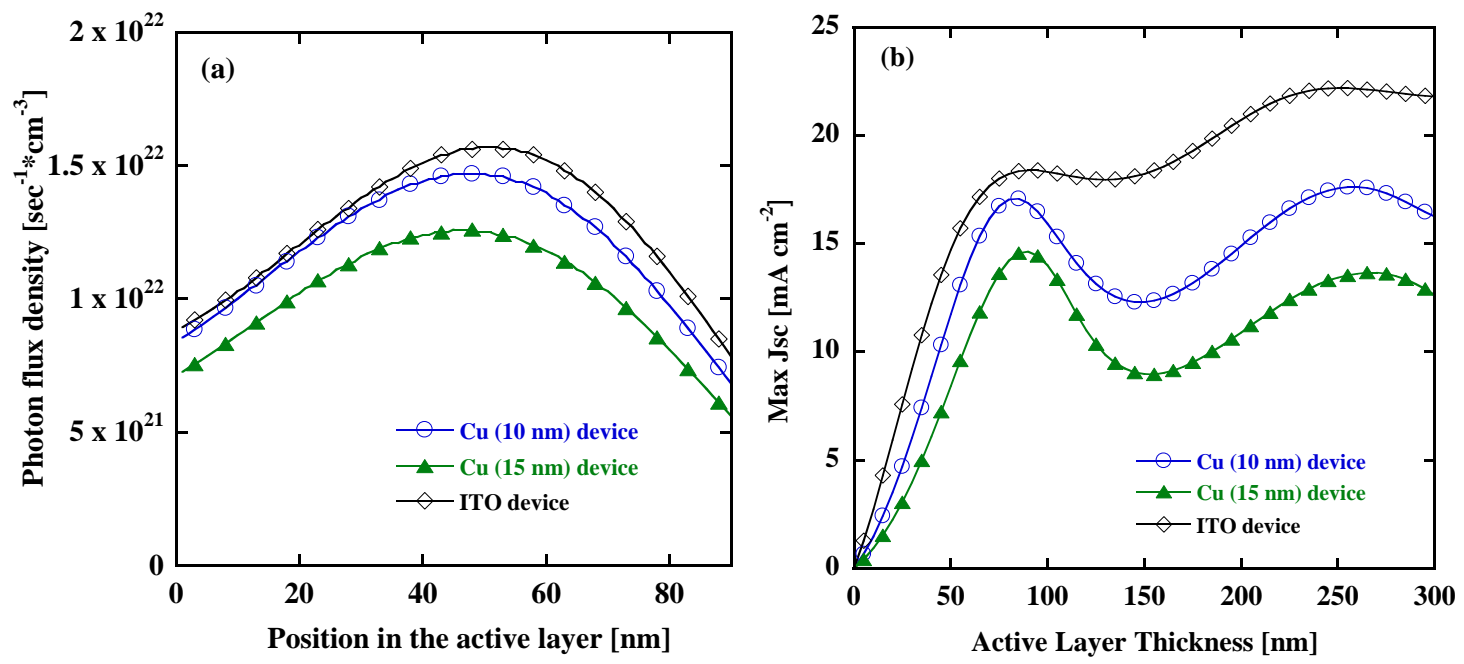

Figure 3.(a) The absorbed photon flux density as a function of position in the active layer for the $\mathrm{Cu}$ film based PSCs under AM 1.5 G illumination. The distribution inside the ITO electrode device is also included for comparison. (b) The dependence of calculated $J_{s c}$ on the active layer thickness for three types of devices under AM 1.5 G illumination.

To quantitatively determine the light harvest inside the ITO-free devices, the measurement of external quantum efficiency (EQE) as a function of the incident light wavelength were carried out, and the results was depicted in Fig.4a. The data of the ITO control devices are also included for comparison. As can be clearly seen, the $\mathrm{Cu}(10 \mathrm{~nm})$ device shows a maximum EQE value of $69.4 \%$ at $680 \mathrm{~nm}$, while the ITO based device shows a peak value of $77.0 \%$ at $635 \mathrm{~nm}$. Moreover, the long wavelength photoresponse of the $\mathrm{Cu}$ device is about $30 \mathrm{~nm}$ red-shifted relative to the ITO based device. The calculated Jsc values obtained by integration of the EQE data with the AM $1.5 \mathrm{G}$ solar spectra were $14.6 \mathrm{~mA} \mathrm{~cm}^{-2}$ for Cu-based device and $17.0 \mathrm{~mA} \mathrm{~cm}^{-2}$ for ITO based device, respectively, which are in good agreement with the deduced values (see Fig. 2). Despite the observed relatively lower $J_{s c}$, the photoresponse of the $\mathrm{Cu}$ device in the long wavelength region between 730 and $800 \mathrm{~nm}$ can outperform that of the ITO device (see Fig. 4a).
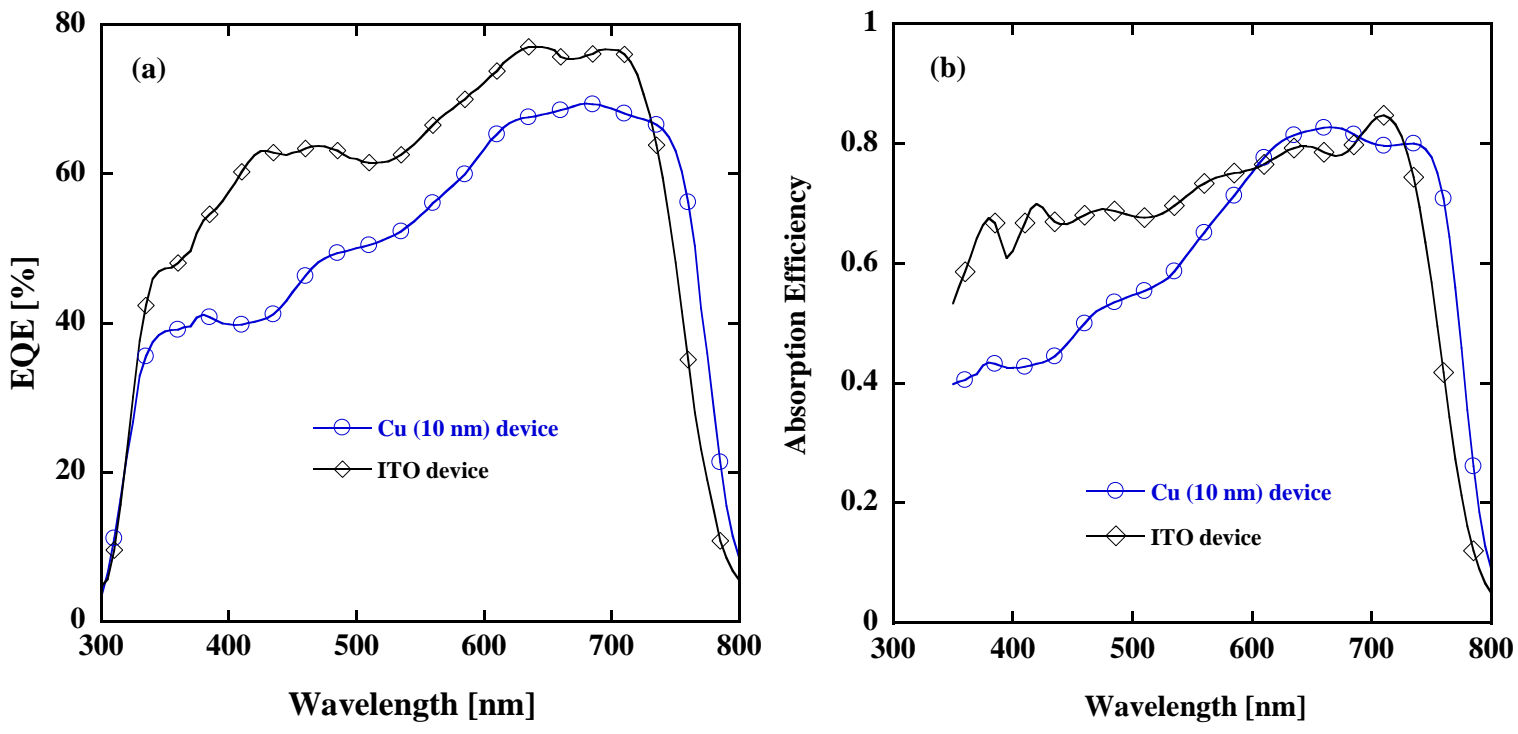


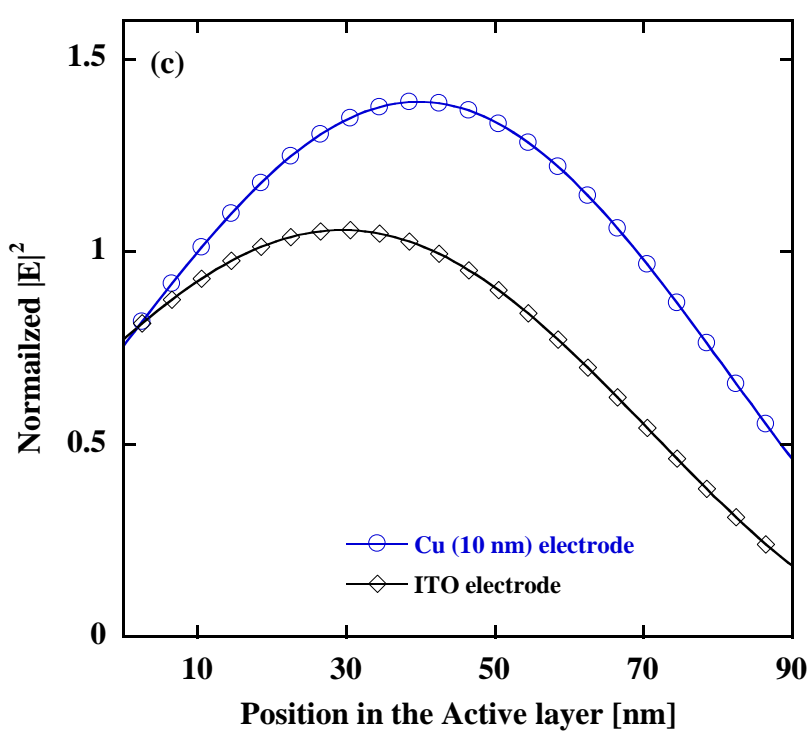

Figure 4.(a) the measured EQE characteristics for the $\mathrm{Cu}$ electrode and ITO electrode devices. (b) the calculated absorption efficiency for the $\mathrm{Cu}$ electrode and ITO electrode devices. (c) the distribution of the normalized electric field intensity of the incident light with a wavelength of $750 \mathrm{~nm}$ inside the devices.

To confirm the abovementioned experimental results that the $\mathrm{Cu}$ device delivery higher photoresponse(EQE) in the long wavelength region, the light absorption efficiency within the photoactive layer of the devices was calculated using one-dimensional transfer matrix formalism[23]. As shown in Fig. $4 \mathrm{~b}$, as compared to the ITO device, the absorption edge of the $\mathrm{Cu}$ device shows a red shift of $30 \mathrm{~nm}$ and comparable absorption efficiency over a broad wavelength range between 600 $\mathrm{nm}$ and $800 \mathrm{~nm}$. Especially, in the region between $620 \mathrm{~nm}$ and $750 \mathrm{~nm}$, the average absorption efficiency of the $\mathrm{Cu}$ device is found to be around $80 \%$, which is obviously higher than the far-field transmittance of the $\mathrm{Cu}$ film ( $72 \%$, see Figure. 1), implying the formation of a resonant optical microcavity between the opaque back contact mirror $\left(\mathrm{MoO}_{3} / \mathrm{Al}\right.$ electrode) and the transparent $\mathrm{Cu}$ film with high reflectance[15]. As a result, the incident light transmits through the $\mathrm{Cu}$ electrode and travel around between the electrodes, resulting in enhanced light harvesting at the region near the absorption edge of the photoactive layer.

As the light harvesting is in direct proportion with the field intensity of the incident light, its enhancement in the $\mathrm{Cu}$ device in this spectral region and the observed red shift of absorption edge can be also understood by examining the field intensity within the photoactive layer. Fig. 4c depicts the distribution of the normalized electric field intensity of the incident light with a wavelength of $750 \mathrm{~nm}$, at which the spectral absorption is weak, inside both type devices. As can be clearly seen, compared to the ITO device, the $\mathrm{Cu}$ device shows stronger electric field in entire photoactive layer, demonstrating an obvious coherent light trapping effect and responsible for the red shift in the EQE around the absorption edge (Fig. $4 \mathrm{~b}$ ).

As changing the electrode in organic solar cells may has a dramatic effect on the recombination kinetics, we further utilized transient photovoltage (TPV) technique to investigate the carrier recombination dynamics in the devices. Fig. 5 presents the TPV measurement results for a Cu device and an ITO device, which was obtained under a light bias with an intensity of $100 \mathrm{~mW} \mathrm{~cm}^{-2}$ that is similar to the actual working condition. The transient photovoltage of the $\mathrm{Cu}$ device can be described by a mono-exponential decay course that responds to the bimolecular recombination of excess carriers generated by excitation of the pulse laser, following $\delta V=V_{1} \exp (-t / \tau)$, while TPV of the ITO device can only be fitted through a double-exponential decay course following $\delta V=V_{1} \exp \left(-t / \tau_{1}\right)+V_{2} \exp \left(-t / \tau_{2}\right)$. The extracted charge carrier lifetime $(\tau)$ is 31.0 us 
for the Cu device, 45.7 us $\left(\tau_{1}\right)$ and 196 us $\left(\tau_{2}\right)$ for the ITO device. The faster TPV decay signal in the $\mathrm{Cu}$ device may result from more rapid charge extraction, considering its smaller photocurrent compared with the ITO device. For the ITO device, the faster decay course is likely due to bimolecular recombination, while the slower decay course may due to additional trap-assisted recombination processes following the Shockley-Reed-Hall model[24]. Therefore, the charge recombination kinetics in the $\mathrm{Cu}$ device is dominated by bimolecular recombination process, and the loss of charge carriers can be further minimized if more desired active layer morphology is reached.[25]

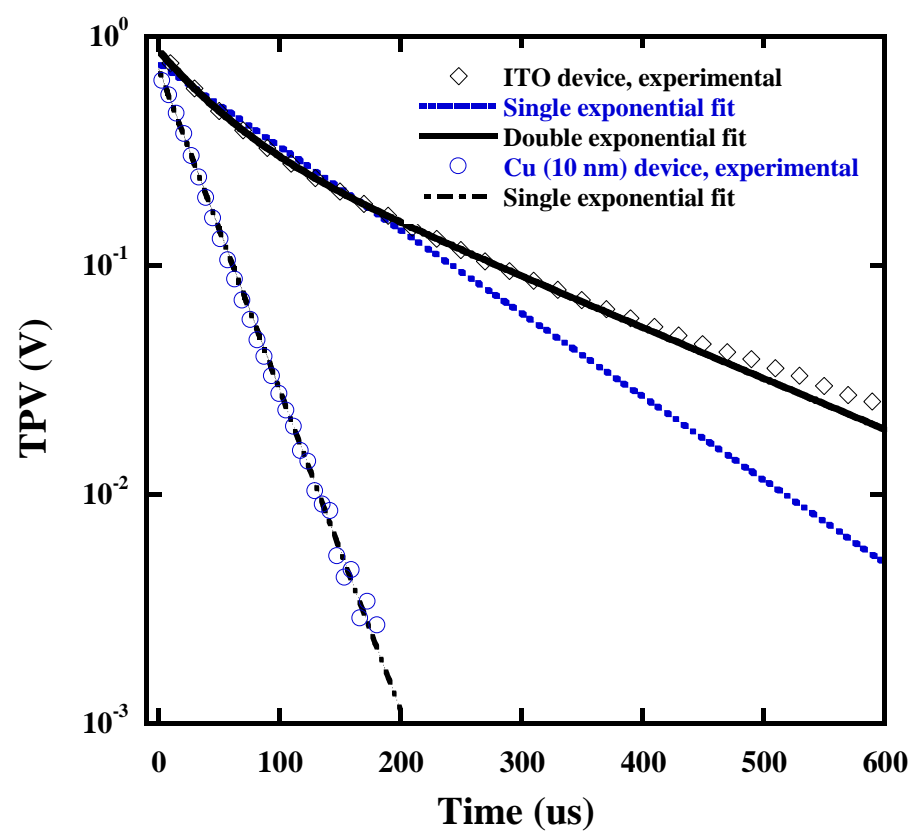

Fig.5.Thetransient photovoltage measurement for the devices based on different electrode, tested under a light intensity of $100 \mathrm{~mW} \mathrm{~cm}^{-2}$ as a bias. The dashed line represents the best single exponential fitting for the $\mathrm{Cu}$ device, while the dished-dot line and solid line represent the best single, double exponential fitting for the ITO device, respectively.

\section{Conclusions}

In conclusion, highly efficient ITO-free polymer solar cells were demonstrated on thermally evaporated $\mathrm{Cu}$ ultrathin film electrode. The $\mathrm{Cu}$ electrodes showed high transmittance in the visible region of the spectrum. Despite its moderate far-field transmittance in near NIR region, the actual absorption efficiency can be effectively enhanced through metal resonant microcavity formed between the opaque back metal mirror and the transparent $\mathrm{Cu}$ film with high reflectance. The device performance of the $\mathrm{Cu}$ film based device were comparable to the reference device by introducing of an electrode interfacial layer, resulting in a high power efficiency of $8.21 \%$, one of the highest values reported to date. Thanks to the very good optical, electrical properties and their low-cost fabrication, the obtained $\mathrm{Cu}$-based electrodes can be applicable to many other donor material systems and may become a promising alternative to ITO-based substrate for polymer solar cells.

Author Contributions: Q.L and G.L designed and directed the research. Q.L and G.L provided the device, analyzed all the data and write the manuscript. G.L contributed to optical modeling and analyzed the data. X.C help to improve the device performance. H.W is responsible for the supervision and revising the manuscript.

Funding: This research was funded by National Natural Science Foundation of China (Nos. 51511130077, 51521002, and 61775061) and Natural Science Foundation of Guangdong University of Petrochemical Technology (Nos.2017rc20). 
Conflicts of Interest: The authors declare no conflict of interest.

\section{References}

1. Inganäs, O. Organic photovoltaics over three decades. Advanced materials 2018, 30, 1800388.

2. Fan, B.; Zhang, D.; Li, M.; Zhong, W.; Zeng, Z.; Ying, L.; Huang, F.; Cao, Y. Achieving over 16\% efficiency for single-junction organic solar cells. Science China Chemistry 2019, 62, 746-752.

3. Yuan, J.; Zhang, Y.; Zhou, L.; Zhang, G.; Yip, H.-L.; Lau, T.-K.; Lu, X.; Zhu, C.; Peng, H.; Johnson, P.A. Single-junction organic solar cell with over $15 \%$ efficiency using fused-ring acceptor with electron-deficient core. Joule 2019, 3, 1140-1151.

4. $\quad$ Zheng, Z.; Hu, Q.; Zhang, S.; Zhang, D.; Wang, J.; Xie, S.; Wang, R.; Qin, Y.; Li, W.; Hong, L. A Highly Efficient Non-Fullerene Organic Solar Cell with a Fill Factor over 0.80 Enabled by a Fine-Tuned Hole-Transporting Layer. Advanced materials 2018, 30, 1801801.

5. He, Z.; Zhong, C.; Su, S.; Xu, M.; Wu, H.; Cao, Y. Enhanced power-conversion efficiency in polymer solar cells using an inverted device structure. Nature Photonics 2012, 6, 593-597.

6. Yan, T.; Song, W.; Huang, J.; Peng, R.; Huang, L.; Ge, Z. 16.67\% Rigid and 14.06\% Flexible Organic Solar Cells Enabled by Ternary Heterojunction Strategy. Advanced materials 2019, 1902210.

7. Meng, L.; Zhang, Y.; Wan, X.; Li, C.; Zhang, X.; Wang, Y.; Ke, X.; Xiao, Z.; Ding, L.; Xia, R. Organic and solution-processed tandem solar cells with 17.3\% efficiency. Science 2018, 361, 1094-1098.

8. $\quad$ Rong, Q.; Zhao, J.; Yu, H.; Li, N.; Zhang, Q.; Yuan, D.; Liu, W.; Zheng, D.; Gao, X.; Shui, L. Light Manipulating Electrode based on High Optical Haze Aluminum-Doped Zinc Oxide for Highly Efficient Indium-Tin-Oxide Free Organic Solar Cells Over 13\% Efficiency. Journal of Materials Chemistry C 2019.

9. Park, J.H.; Han, S.; Kim, D.; You, B.K.; Joe, D.J.; Hong, S.; Seo, J.; Kwon, J.; Jeong, C.K.; Park, H.J. Plasmonic-Tuned Flash $\mathrm{Cu}$ Nanowelding with Ultrafast Photochemical-Reducing and Interlocking on Flexible Plastics. Advanced Functional Materials 2017, 27, 1701138.

10. Jeong, S.; Cho, H.; Han, S.; Won, P.; Lee, H.; Hong, S.; Yeo, J.; Kwon, J.; Ko, S.H. High efficiency, transparent, reusable, and active PM2. 5 filters by hierarchical Ag nanowire percolation network. Nano letters 2017, 17, 4339-4346.

11. Lu, S.; Sun, Y.; Ren, K.; Liu, K.; Wang, Z.; Qu, S. Recent development in ITO-free flexible polymer solar cells. Polymers 2017, 10, 5.

12. Stec, H.M.; Williams, R.J.; Jones, T.S.; Hatton, R.A. Ultrathin Transparent Au Electrodes for Organic Photovoltaics Fabricated Using a Mixed Mono-Molecular Nucleation Layer. Advanced Functional Materials 2011, 21, 1709-1716.

13. Sergeant, N.P.; Hadipour, A.; Niesen, B.; Cheyns, D.; Heremans, P.; Peumans, P.; Rand, B.P. Design of transparent anodes for resonant cavity enhanced light harvesting in organic solar cells. Advanced materials 2012, 24, 728-732.

14. Chen, Y.; Shen, L.; Yu, W.; Long, Y.; Guo, W.; Chen, W.; Ruan, S. Highly efficient ITO-free polymer solar cells based on metal resonant microcavity using WO 3 /Au/WO 3 as transparent electrodes. Organic Electronics 2014, 15, 1545-1551.

15. Han, D.; Lee, S.; Kim, H.; Jeong, S.; Yoo, S. Cathodic multilayer transparent electrodes for ITO-free inverted organic solar cells. Organic Electronics 2013, 14, 1477-1482.

16. Jung, G.H.; Hong, K.; Dong, W.J.; Kim, S.; Lee, J.L. BCP/Ag/MoO3 Transparent Cathodes for Organic Photovoltaics. Advanced Energy Materials 2011, 1, 1023-1028. 
17. Ham, J.; Lee, J.L. ITO Breakers: Highly Transparent Conducting Polymer/Metal/Dielectric (P/M/D) Films for Organic Solar Cells. Advanced Energy Materials 2015, 4.

18. Li, K.; Zhen, H.; Niu, L.; Fang, X.; Zhang, Y.; Guo, R.; Yu, Y.; Yan, F.; Li, H.; Zheng, Z. Full-solution processed flexible organic solar cells using low-cost printable copper electrodes. Advanced materials 2014, 26, 7271-7278.

19. Hutter, O.S.; Hatton, R.A. A hybrid copper:tungsten suboxide window electrode for organic photovoltaics. Advanced materials 2015, 27, 326-331.

20. Huang, F.; Wu, H.; Deli Wang; Yang, W.; Cao, Y. Novel Electroluminescent Conjugated Polyelectrolytes Based on Polyfluorene. Chemistry of Materials 2004, 16, 708-716.

21. He, Z.; Xiao, B.; Liu, F.; Wu, H.; Yang, Y.; Xiao, S.; Wang, C.; Russell, T.P.; Cao, Y. Single-junction polymer solar cells with high efficiency and photovoltage. Nature Photonics 2015, 9.

22. Luo, G.; Cheng, X.; He, Z.; Wu, H.; Cao, Y. High-performance inverted polymer solar cells based on thin copper film. Journal of Photonics for Energy 2014, 5, 057206.

23. Pettersson, L.A.A.; Roman, L.S.; Inganäs, O. Modeling photocurrent action spectra of photovoltaic devices based on organic thin films. Journal of Applied Physics 1999, 86, 487-496.

24. Shockley, W.; Read, W.T. Statistics of the recombinations of holes and electrons. 1952, 87, 835-842.

25. Yan, J.; Liang, Q.; Liu, K.; Miao, J.; Hui, C.; Sha, L.; He, Z.; Wu, H.; Wang, J.; Yong, C. Optimized Phase Separation and Reduced Geminate Recombination in High Fill Factor Small-Molecule Organic Solar Cells. Acs Energy Letters 2016, 2, 14-21. 